Chinese Journal of Organic Chemistry

NOTE

\title{
1'-芳甲基二茂铁联烯的合成
}

$\begin{array}{clll}\text { 王晨君 } & \text { 王 } & \text { 晗甫超张开新 } \\ \text { 韩飞 } & \text { 李保国* } & \text { 陈树峰* }\end{array}$

(内蒙古大学化学化工学院 内蒙古自治区精细有机合成重点实验室 呼和浩特 010021 )

\begin{abstract}
摘要 设计了新型 1 -芳甲基二茂铁联烯衍生物的合成方法, 该方法以方便易得的二茂铁为底物，通过傅氏酰基化、还 原、乙酰化、乙炔化及改进的 Crabbé 反应等过程，合成了六种目标化合物. 并通过核磁共振氢谱、碳谱及高分辨质谱 对其结构进行了表征.
\end{abstract}

关键词 二茂铁; 联烯; 1'-芳甲基二茂铁联烯; 合成

\section{Synthesis of 1'-Arylmethyl-1-(1,2-dienyl)ferrocenes}

\author{
Wang, Chenjun Wang, Han Ma, Chao Zhang, Kaixin \\ Han, Fei Li, Baoguo* Chen, Shufeng* \\ (Inner Mongolia Key Laboratory of Fine Organic Synthesis, Department of Chemistry and Chemical Engineering, \\ Inner Mongolia University, Hohhot 010021)
}

\begin{abstract}
A convenient procedure for synthesizing 1'-arylmethyl-1-(1,2-dienyl)ferrocene derivatives was explored. Using commercially available ferrocene as the starting material, the six target compounds were obtained in five steps via Friedel-Crafts acylation, reduction, acetylation, acetylene formation and modified Crabbé reaction. The structures of the obtained allenes were characterized by ${ }^{1} \mathrm{H}$ NMR and ${ }^{13} \mathrm{C}$ NMR and HRMS.
\end{abstract}

Keywords ferrocene; allene; 1'-arylmethyl-1-(1,2-dienyl)ferrocene; synthesis

自从 20 世纪 50 年代首次发现二茂铁以来 ${ }^{[1]}$, 二茂 铁由于其独特的结构特征、化学和热稳定性以及电化学 特性受到了化学家的广泛关注 ${ }^{[24]}$. 这些特性在材料化

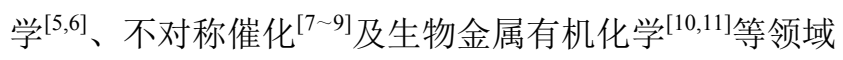
有着重要应用. 此外, 研究表明, 一些特定结构的含二 茂铁基团的化合物具有多种生物生理活性, 如抗肿瘤、 抗炎、杀菌、抗贫血等 ${ }^{[12,13]}$. 因此, 设计和合成结构新 颖的二茂铁衍生物仍是有机化学家的重要研究领域.

近些年, 联烯由于其独特的结构和化学特性, 受到 了有机化学家的格外关注 ${ }^{[14 \sim 19]}$. 研究表明, 联烯可以表 现出一些特有的反应活性、进行各种各样的转化, 从而 方便地构筑许多结构复杂的有机化合物. 因此, 设计和 合成结构新颖的联烯化合物是该领域的研究热点之一. 在过去几十年中, 化学家发展了许多联烯的合成方 法 ${ }^{[2024]}$. 其中, Crabbé 反应是以简单易得化合物为原料
合成单取代联烯的经典方法 ${ }^{[25]}$. 近期, 麻生明课题组对 Crabbé 反应进行了一系列重要改进, 开创性地发展了各

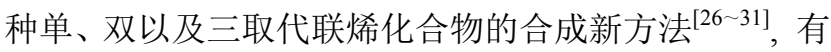
力地促进了联烯化学的发展.

此外，如果将二茂铁基团导入到联烯结构中，很可 能会改变它们的固有性质, 得到一些性质特殊的新奇的 二茂铁联烯化合物. 然而, 文献中关于二茂铁联烯化合 物的合成基本上没有报道. 基于我们课题组对二茂铁化 学及联烯化学的研究兴趣, 近期我们报道了双取代二茂 铁联烯 ${ }^{[32]}$ 及 $1^{\prime}$-酰基二茂铁联烯 ${ }^{[33]}$ 化合物的合成. 作为 该工作的延续，本文报道了一系列 1 '-芳甲基二茂铁联 烯化合物的合成方法. 该方法以方便易得的二茂铁为原 料, 通过傅氏酰基化、还原、乙酰化、乙炔化及改进的 Crabbé 反应等步骤, 方便地得到了目标化合物, 合成过 程如 Scheme 1 中路线 B 所示.

*E-mail: shufengchen@imu.edu.cn; baoguol@sohu.com

Received February 8, 2015; revised March 8, 2015; published online March 15, 2015.

Project supported by the National Natural Science Foundation of China (No. 21262023) and the Natural Science Foundation of Inner Mongolia of China (No. 2014JQ02).

国家自然科学基金(No. 21262023)和内蒙古自治区杰出青年培育基金(No. 2014JQ02)资助项目. 


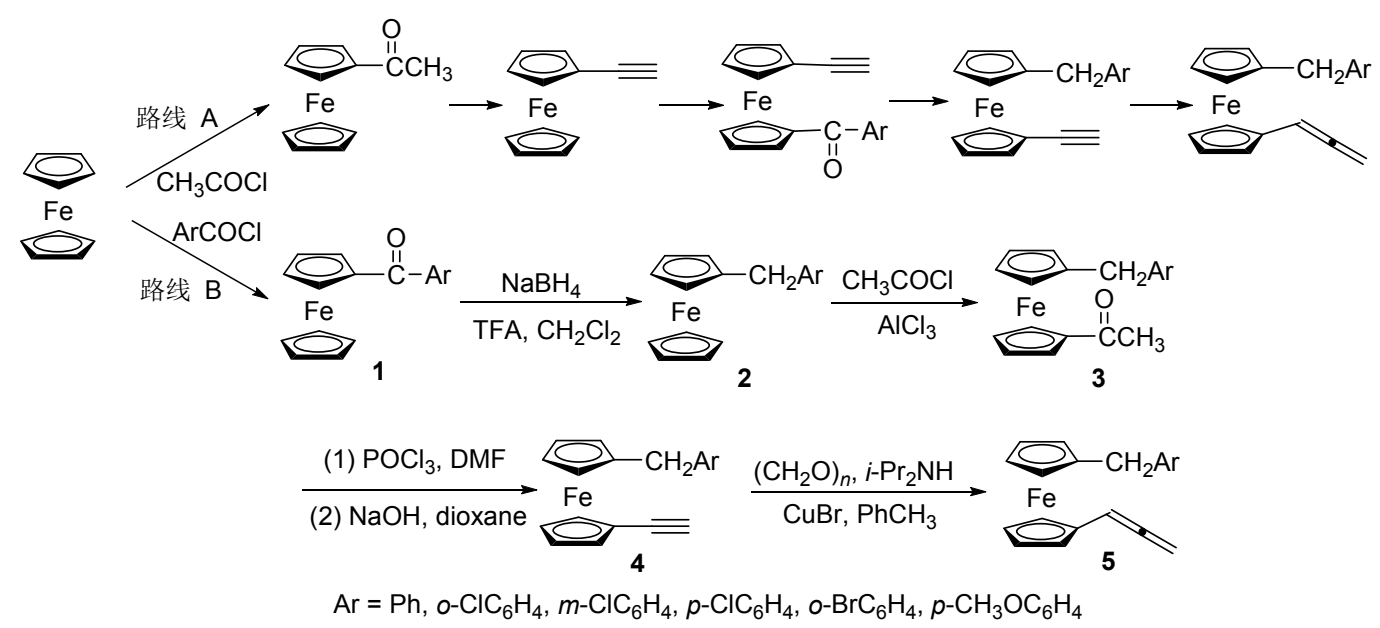

图式 1 合成路线设计

Scheme 1 Design the synthetic route

\section{1 结果与讨论}

\section{1 合成路线设计}

为了获得最简便的合成路线来合成目标化合物, 我 们首先对课题组之前报道的 $1^{\prime}$-酰基二茂铁联烯化合物 的酰基进行还原, 试图直接合成 1'-芳甲基二茂铁联烯. 但遗憾的是, 我们尝试了各种还原方法, 都无法得到目 标化合物, 结果表明, 酰基被还原的同时, 联烯基团也 无法保留. 该结果促使我们继续设计更加合理的路线来 合成该类新颖的 1 '芳甲基二茂铁联烯化合物。根据我 们的经验, 我们以二茂铁为原料, 设计了两条可能的合 成路线, 如 Scheme 1 所示. 其中, 路线 A 首先对二茂铁 进行乙酰化, 进而将乙酰基转变为乙炔基, 接下来在二 茂铁的另一个环戊二烯基环上进行芳甲酰化, 进而将其 还原为芳甲基, 最后通过 Crabbé 反应将乙炔基转变为 联烯. 但令人遗憾的是, 在我们试图将二茂铁乙炔进行 芳甲酰化时，无论何种条件，都无法在保留乙炔基的同 时得到芳甲酰化产物.

接下来, 我们又改变合成策略, 同样以二茂铁为原 料，首先进行芳甲酰化反应，接着将其还原为芳甲基， 然后再进行乙酰化, 进而将乙酰基转变为乙炔基, 最后 通过改进的 Crabbé 反应将乙炔基转化为联烯基团，该 合成路线如 Scheme 1 路线 B 所示. 令我们欣喜的是, 路 线 $\mathrm{B}$ 是可行的, 最终我们顺利地得到了目标化合物 1'芳甲基二茂铁联烯.

\section{2 芳甲酰基二茂铁(1)和芳甲基二茂铁(2)的合成}

为了实现我们的目的，首先通过经典的傅氏酰基化 反应合成了一系列芳甲酰基二茂铁, 研究表明, 各种芳 甲酰氯与二茂铁均能很好地反应, 以较高产率得到酰化 产物. 接下来, 我们考察了不同还原剂对所得酰化产物
的还原效果. 研究发现, 用 $\mathrm{NaBH}_{4}$-三氟乙酸还原体系在 二氯甲烷作溶剂时室温下即能高效地将羰基还原为亚 甲基，该方法与 Clemmensen 还原相比，避免了锌录齐 的使用，而且条件温和、反应后处理方便，实验结果见 表 1 . 值得一提的是，当苯环上有供电子基甲氧基存在 时，只能以中等产率得到相应的还原产物.

\subsection{1-乙酰基-1'-芳甲基二茂铁(3)和 1-乙炔基-1'-芳甲 基二茂铁(4)的合成}

合成上述芳甲基二茂铁化合物后，接下来的任务是 向二茂铁的另一个环上引入乙炔基. 首先, 我们将苯甲 基二茂铁与乙酰氯在 $\mathrm{AlCl}_{3}$ 催化下进行傅氏酰基化作为 模型反应，考察影响反应的因素. 我们担心的是，由于 苯环的存在，可能导致乙酰化反应既可以发生在二茂铁 的环戊二烯基环上, 又可以发生在苯环上. 为了得到单 一目标化合物 3 , 我们进行了大量实验，最终发现严格 控制反应温度于 $-15{ }^{\circ} \mathrm{C}$, 缓慢向芳甲基二茂铁的无水 二氯甲烷溶液中滴加预先制备好的乙酰氯一三氯化铝混 合液，高产率高选择性地得到了目标化合物 $\mathbf{3 a}$, 而且没 有检测到苯环上乙酰化的产物。接下来，我们将该方法 应用到其它芳甲基二茂铁底物上，也得到了令人满意的 结果(表 2). 需要说明的是, 当苯环上有供电子基甲氧基 存在时, 乙酰化产物的产率相对较低, 这是因为在该反 应中苯环上的乙酰化是无法避免的.

接下来, 参考之前的研究结果 ${ }^{[33]}$, 我们考察了 1-乙 炔基-1'-芳甲基二茂铁(4)的合成. 结果表明，对于上述 制备的不同底物，均能得到较好的结果，如表 2 所示. 需要指出的是, 该反应所需的 $\mathrm{POCl}_{3}$ 和 $\mathrm{DMF}$ 对反应影 响较大, 需要在使用前纯化处理, 否则将大大降低反应 收率. 另外, 在用 $\mathrm{NaOH}$ 溶液处理时, 一定要迅速加入 预先加热好的 $90{ }^{\circ} \mathrm{C}$ 的 $\mathrm{NaOH}(0.5 \mathrm{~mol} / \mathrm{L})$ 溶液, 这是获 
表 1 芳甲酰基二茂铁和芳甲基二茂铁的合成

Table 1 Synthesis of arylcarbonyl ferrocenes and arylmethyl ferrocenes

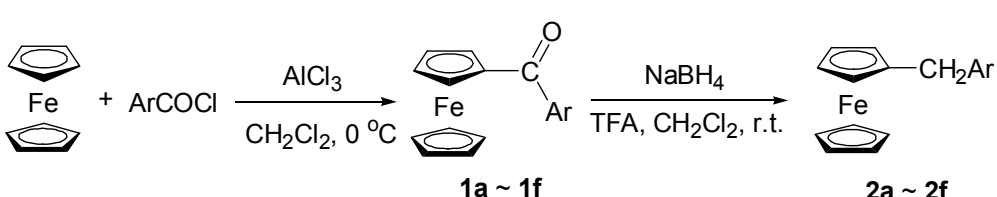

1a $\sim 1 f \quad 2 a \sim 2 f$

\begin{tabular}{|c|c|c|c|c|}
\hline Entry & Product 1 & Yield $^{a} / \%$ of $\mathbf{1}$ & Product 2 & Yield $^{a} / \%$ of 2 \\
\hline 1 & & 75 & & 75 \\
\hline 2 & & 68 & & 61 \\
\hline 3 & & 81 & & 60 \\
\hline 4 & & 75 & & 74 \\
\hline 5 & & 80 & & 74 \\
\hline \multirow[t]{2}{*}{6} & & 58 & & 42 \\
\hline & $1 \mathrm{f}$ & & $2 f$ & \\
\hline
\end{tabular}

${ }^{a}$ Yield of isolated product after chromatography.

得较理想反应结果的前提条件.

\subsection{1'-芳甲基二茂铁联烯(5)的合成}

成功合成上述 1-乙炔基-1'-芳甲基二茂铁化合物 $\mathbf{4 a} \sim \mathbf{4 f}$ 后, 我们利用改进的 Crabbé 反应将其转化为联烯 化合物. 首先, 参考之前的研究方法 ${ }^{[33]}$, 以 $\mathrm{CuI}$ 作催化 剂, 二异丙胺作碱, 化合物 $\mathbf{4 a}$ 与多聚甲醛在甲苯中回流 $6 \mathrm{~h}$, 以 $35 \%$ 的产率得到了目标化合物. 为了提高化合物 5a 的产率, 我们又详细考察了催化剂、碱以及溶剂对该 反应的影响. 研究发现, 当以 $\mathrm{CuBr}(80 \mathrm{~mol} \%)$ 作催化剂、 二异丙胺 $(200 \mathrm{~mol} \%)$ 作碱时，化合物 $\mathbf{4 a}$ 与多聚甲醛在甲 苯中回流 $1 \mathrm{~h}$, 可以得到较高收率的联烯化合物 $\mathbf{5 a}$. 接
下来, 在相同的反应条件下，考察了各种 1-乙炔基-1'-芳 甲基二茂铁化合物转化为联烯的效率，获得了令人满意 的结果(表 3).

\section{2 结论}

本文以二茂铁为底物，通过傅氏酰基化、还原、乙 酰化、乙炔化及改进的 Crabbé 反应等过程顺利地合成 了一系列 1 -芳甲基二茂铁联烯化合物. 合成方法操作 简便，处理方便，为 1'-芳甲基二茂铁联烯的合成提供了 一条可行的途径. 
表 2 1-乙酰基-1'-芳甲基二茂铁和 1-乙炔基-1'-芳甲基二茂铁的合成

Table 2 Synthesis of 1-acetyl-1'-arylmethyl ferrocenes and 1-ethhynyl-1'-arylmethyl ferrocenes

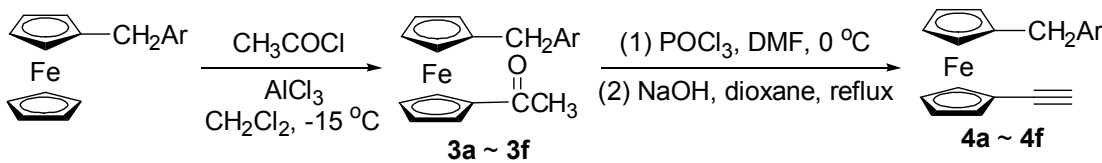

Entry

${ }^{a}$ Yield of isolated product after chromatography.

\section{3 实验部分}

\section{1 仪器与试剂}

Advanced Brucker-500 MHz 核磁共振仪(TMS 为内 标, $\mathrm{CDCl}_{3}$ 为溶剂); Bruker ultrafleXtreme MALDI-TOF/ TOF 高分辨质谱仪. 所用试剂均为市售分析纯试剂, 溶 剂的干燥按常规方法进行. 柱层析使用 $200 \sim 300$ 目硅
胶，展开剂为石油醚 $\left(60 \sim 90{ }^{\circ} \mathrm{C}\right)$ 和乙酸乙酯.

\section{2 实验方法}

\section{2 .1 化合物 1 的合成方法}

在 $0{ }^{\circ} \mathrm{C}$ 下，将芳甲酰氯 $(0.118 \mathrm{~mol})$ 缓慢地滴加到无 水三氯化铝 $(0.118 \mathrm{~mol})$ 和 $135 \mathrm{~mL}$ 无水 $\mathrm{CH}_{2} \mathrm{Cl}_{2}$ 形成的 混合液中, 搅拌 $15 \mathrm{~min}$, 直到三氯化铝基本溶解. 然后 
表 3 1'-芳甲基二茂铁联烯的合成

Table 3 Synthesis of 1'-arylmethyl-1-(1,2-dienyl)ferrocenes
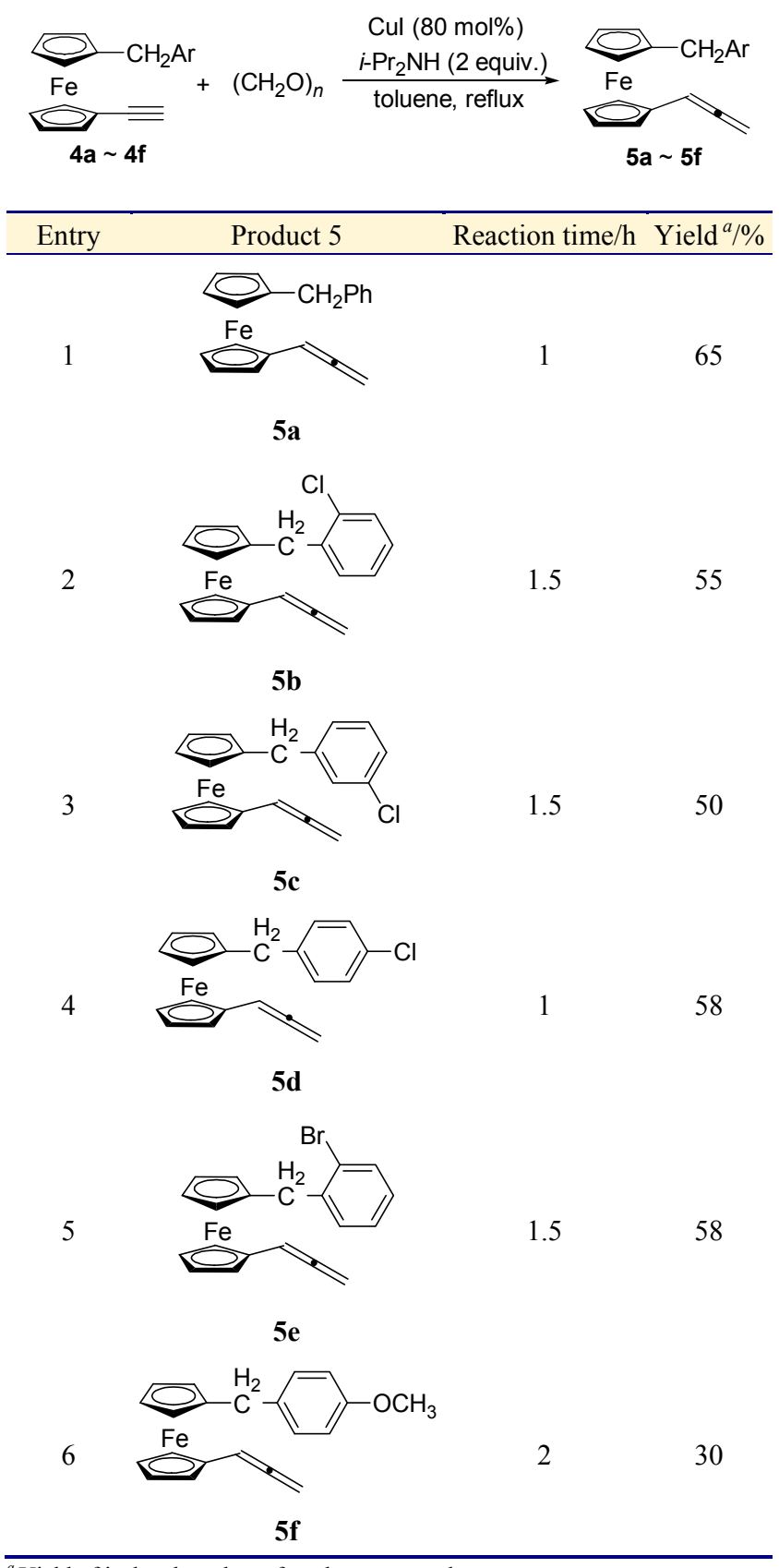

${ }^{a}$ Yield of isolated product after chromatography.

在 $0{ }^{\circ} \mathrm{C}$ 下将上述制备好的混合液缓慢滴加到溶有二茂 铁 $(0.107 \mathrm{~mol})$ 的 $335 \mathrm{~mL} \mathrm{CH} \mathrm{Cl}_{2}$ 溶液中, 于 $0 \sim 10{ }^{\circ} \mathrm{C}$ 下 搅拌 $45 \mathrm{~min}$, 再于室温搅拌反应 $3 \mathrm{~h}$. 反应完毕后, 将反 应混合液倒入冰水中分解, 分出有机相, 水相用 $\mathrm{CH}_{2} \mathrm{Cl}_{2}$ 萃取, 合并有机相, 水洗, 有机层用无水硫酸镁干燥, 过滤, 减压蒸馏蒸去溶剂, 柱层析 $[V$ (石油醚) : $V($ 乙酸 乙酯) $=10 ：$ ] 纯化得到目标产物 $\mathbf{1 a} \sim \mathbf{1 f}$.

\section{2 .2 化合物 2 的合成方法}

向 $250 \mathrm{~mL}$ 反应瓶中依次加入芳甲酰基二茂铁
$(0.016 \mathrm{~mol}) 、 35 \mathrm{~mL}$ 三氟乙酸(TFA)和 $17.5 \mathrm{~mL} \mathrm{CH}_{2} \mathrm{Cl}_{2}$, 搅拌均匀后, 在 $30 \mathrm{~min}$ 内分批加入 $2.7 \mathrm{~g} \mathrm{NaBH}_{4}$, 加毕, 在室温下搅拌反应 $45 \mathrm{~min}$. TLC 监测反应完毕, 向反应 液中加入 $105 \mathrm{~mL} \mathrm{CH}_{2} \mathrm{Cl}_{2}$ 稀释, 再加入 $2 \mathrm{~mol} / \mathrm{L}$ 的 $\mathrm{NaOH}$ 溶液碱化至碱性 $(\mathrm{pH}=10)$. 混合液用 $\mathrm{CH}_{2} \mathrm{Cl}_{2}$ 萃取 3 次, 有机相用无水 $\mathrm{MgSO}_{4}$ 干燥, 过滤, 减压蒸馏蒸去溶剂, 柱层析(石油醚)纯化得到目标产物 $\mathbf{2 a} \sim 2 \mathbf{f}$.

\section{2 .3 化合物 3 的合成方法}

向 $250 \mathrm{~mL}$ 反应瓶中加入 $0.020 \mathrm{~mol}$ 无水 $\mathrm{AlCl}_{3}$ 和 24 $\mathrm{mL}$ 无水 $\mathrm{CH}_{2} \mathrm{Cl}_{2}$ 加入到单口瓶中, 冰盐浴冷却至一 $15{ }^{\circ} \mathrm{C}$, 搅拌下缓慢滴加 $0.018 \mathrm{~mol} \mathrm{CH}_{3} \mathrm{COCl}$ 和 $16 \mathrm{~mL}$ 无 水 $\mathrm{CH}_{2} \mathrm{Cl}_{2}$ 混合溶液, 搅拌直至 $\mathrm{AlCl}_{3}$ 溶解, 备用.

向另一 $250 \mathrm{~mL}$ 三颈瓶中依次加入 $0.017 \mathrm{~mol}$ 芳甲基 二茂铁 2 和 $16 \mathrm{~mL}$ 无水 $\mathrm{CH}_{2} \mathrm{Cl}_{2}$, 冰盐浴冷却下缓慢滴加 上述制备好的乙酰氯一三氯化铝混合液. 滴毕, 继续摚 拌直至无氯化氢气体放出. 将反应混合液倒入冰水中分 解, 分出有机相, 水相用 $\mathrm{CH}_{2} \mathrm{Cl}_{2}$ 萃取, 合并有机相, 依 次用 $5 \%$ 的 $\mathrm{Na}_{2} \mathrm{CO}_{3}$ 水溶液和水分别洗涤有机相, 有机 层用无水硫酸镁干燥, 过滤, 减压蒸馏蒸去溶剂, 柱层 析 $[V($ 石油醚 $) ： V($ 乙酸乙酯 $)=10: 1]$ 纯化得到目标产物 $\mathbf{3 a} \sim \mathbf{3 f}$.

\subsection{4 化合物 $\mathbf{4}$ 的合成方法}

氮气保护下，向 $250 \mathrm{~mL}$ 反应瓶中加入 $13.27 \mathrm{mmol}$ 化合物 3 和 $20 \mathrm{~mL}$ 无水 DMF, 冰水浴冷却下搅拌溶解. 于 $0{ }^{\circ} \mathrm{C}$ 下缓慢滴加 $\mathrm{POCl}_{3}(4 \mathrm{~mL})$ 的 $15 \mathrm{~mL}$ 无水 $\mathrm{DMF}$ 溶 液, 滴毕, 继续摚拌 $2 \mathrm{~h}$. 然后, 向反应液中加入 $100 \mathrm{~mL}$ $20 \%$ 的 $\mathrm{CH}_{3} \mathrm{COONa}$ 水溶液, 继续摚拌 $1.5 \mathrm{~h}$. 反应完毕, 混合液用 $\mathrm{CH}_{2} \mathrm{Cl}_{2}$ 萃取 3 次, 有机相用无水硫酸镁干燥, 过滤, 减压蒸馏蒸去溶剂, 柱层析 $[V$ (石油醚) $: V($ 乙酸 乙酯) $=20 ： 1]$ 纯化得到中间体备用.

氮气保护下, 将上述制备好的中间体 $11.7 \mathrm{mmol}$ 和 $100 \mathrm{~mL}$ 无水 1,4-二氧六环依次加入到 $250 \mathrm{~mL}$ 三口瓶中, 加热至回流后, 立刻加入预先制备的 $62 \mathrm{~mL} 0.5 \mathrm{~mol} / \mathrm{L}$ $90{ }^{\circ} \mathrm{C}$ 的 $\mathrm{NaOH}$ 溶液, 继续回流 $30 \mathrm{~min}$. 将反应液倾入至 冰水中, 盐酸酸化, 水相用 $\mathrm{CH}_{2} \mathrm{Cl}_{2}$ 萃取 3 次, 合并有机 相, 水洗, 有机层用无水硫酸镁干燥, 过滤, 减压蒸馏 蒸去溶剂, 柱层析(石油醚)纯化得到目标产物 $\mathbf{4 a} \sim \mathbf{4 f}$.

$1^{\prime}$-苯甲基二茂铁乙炔(4a)：黄色固体，产率 75\%. ${ }^{1} \mathrm{H}$ NMR (500 MHz, $\left.\mathrm{CDCl}_{3}\right) \delta: 2.78(\mathrm{~s}, 1 \mathrm{H}), 3.70(\mathrm{~s}, 2 \mathrm{H}), 4.12$ (d, $J=10.5 \mathrm{~Hz}, 3 \mathrm{H}), 4.16(\mathrm{~d}, J=4.5 \mathrm{~Hz}, 3 \mathrm{H}), 4.40(\mathrm{~s}, 2 \mathrm{H})$, $7.17(\mathrm{dd}, \quad J=13.5,8.0 \mathrm{~Hz}, 3 \mathrm{H}), 7.23 \sim 7.28(\mathrm{~m}, 2 \mathrm{H}) ;{ }^{13} \mathrm{C}$ NMR $\left(125 \mathrm{MHz}, \mathrm{CDCl}_{3}\right) \delta: 34.69,64.48,69.39,69.61$, $70.48,70.77,72.40,74.10,82.38,88.78,125.98,128.32$, 128.45, 141.48. HRMS (ESI) calcd for $\mathrm{C}_{19} \mathrm{H}_{16} \mathrm{Fe} 300.0601$, found 300.0607 . 
1'-(2-氯苯甲基)二茂铁乙炔 $(\mathbf{4 b})$ : 黄色油状液体, 产 率 $82 \% .{ }^{1} \mathrm{H}$ NMR $\left(500 \mathrm{MHz}, \mathrm{CDCl}_{3}\right) \delta: 2.79(\mathrm{~s}, 1 \mathrm{H}), 3.82$ (s, $2 \mathrm{H}), 4.16 \sim 4.20(\mathrm{~m}, 6 \mathrm{H}), 4.42 \sim 4.43(\mathrm{~m}, 2 \mathrm{H}), 7.07 \sim$ $7.14(\mathrm{~m}, 3 \mathrm{H}), 7.30 \sim 7.32(\mathrm{~m}, 1 \mathrm{H}) ;{ }^{13} \mathrm{C}$ NMR $(125 \mathrm{MHz}$, $\left.\mathrm{CDCl}_{3}\right) \delta: 32.23,64.68,69.68,70.90,72.48,73.70,74.38$, $82.30,87.08,126.78,127.45,129.31,130.27,133.73$, 139.37. HRMS (ESI) calcd for $\mathrm{C}_{19} \mathrm{H}_{15} \mathrm{ClFe}$ 334.0207, found 334.0207 .

$1 '$-(3-氯苯甲基)二茂铁乙炔 $(\mathbf{4 c})$ : 黄色油状液体, 产 率 87\%. ${ }^{1} \mathrm{H}$ NMR (500 MHz, $\left.\mathrm{CDCl}_{3}\right) \delta: 2.79(\mathrm{~s}, 1 \mathrm{H}), 3.66$ (s, 2H), 4.09 (s, 2H), 4.13 4.16 (m, 4H), 4.39 (s, 2H), 7.03 (dd, $J=18.5,7.0 \mathrm{~Hz}, 1 \mathrm{H}), 7.13 \sim 7.18(\mathrm{~m}, 3 \mathrm{H}) ;{ }^{13} \mathrm{C}$ NMR $\left(125 \mathrm{MHz}, \mathrm{CDCl}_{3}\right) \delta: 34.36,64.65,69.43,69.74$, 70.49, 72.46, 74.37, 82.27, 87.83, 126.21, 126.66, 128.58, 129.59, 134.08, 143.55. HRMS (ESI) calcd for $\mathrm{C}_{19} \mathrm{H}_{15} \mathrm{ClFe}$ 334.0207 , found 334.0215 .

1'-(4-氯苯甲基)二茂铁乙炔 $(4 d)$ : 黄色固体, 产率 $53 \%$. ${ }^{1} \mathrm{H}$ NMR (500 MHz, $\mathrm{CDCl}_{3}$ ) $\delta 2.78(\mathrm{~s}, 1 \mathrm{H}), 3.67$ (s, $2 \mathrm{H}), 4.08(\mathrm{~s}, 2 \mathrm{H}), 4.12 \sim 4.20(\mathrm{~m}, 4 \mathrm{H}), 4.40(\mathrm{~s}, 2 \mathrm{H}), 7.07 \sim$ 7.14 (m, 2H), $7.21 \sim 7.25$ (m, 2H); ${ }^{13} \mathrm{C}$ NMR $(125 \mathrm{MHz}$, $\left.\mathrm{CDCl}_{3}\right) \delta: 33.96,64.58,69.38,69.66,70.36,70.80,72.42$, $74.23,82.26,88.22,128.40,129.79,131.72,139.91$. HRMS (ESI) calcd for $\mathrm{C}_{19} \mathrm{H}_{15} \mathrm{ClFe}$ 334.0207, found 334.0216

1'-(2-溴苯甲基)二茂铁乙炔 (4e)：黄色油状液体，产 率 50\%. ${ }^{1} \mathrm{H}$ NMR $\left(500 \mathrm{MHz}, \mathrm{CDCl}_{3}\right) \delta 2.41(\mathrm{~s}, 1 \mathrm{H}), 3.74$ $(\mathrm{s}, 2 \mathrm{H}), 4.15(\mathrm{~s}, 1 \mathrm{H}), 4.20(\mathrm{~s}, 3 \mathrm{H}), 4.50(\mathrm{~s}, 2 \mathrm{H}), 4.76(\mathrm{~s}$, $2 \mathrm{H}), 7.02 \sim 7.14(\mathrm{~m}, 2 \mathrm{H}), 7.17 \sim 7.23(\mathrm{~m}, 1 \mathrm{H}), 7.50 \sim 7.55$ $(\mathrm{m}, 1 \mathrm{H}) ;{ }^{13} \mathrm{C}$ NMR $\left(125 \mathrm{MHz}, \mathrm{CDCl}_{3}\right) \delta: 35.22,69.38$, 69.76, 70.42, 70.73, 72.40, 73.36, 79.85, 88.20, 124.16, 127.46, 127.87, 130.17, 132.66, 140.49. HRMS (ESI) calcd for $\mathrm{C}_{19} \mathrm{H}_{15} \mathrm{BrFe} 377.9703$, found 337.9700 .

1'-(4-甲氧苯甲基)二茂铁乙炔(4f)：黄色固体，产率 $80 \% .{ }^{1} \mathrm{H}$ NMR (500 MHz, $\mathrm{CDCl}_{3}$ ) $\delta: 2.77$ (s, 1H), 3.64 (s, 2H), 3.76 (s, 3H), 4.09 (s, 2H), 4.12 (s, 1H), 4.15 (d, $J=$ $5.0 \mathrm{~Hz}, 3 \mathrm{H}), 4.39$ (s, 2H), 6.81 (dd, $J=8.5,2.5 \mathrm{~Hz}, 2 \mathrm{H})$, 7.09 (dd, $J=15.5,8.5 \mathrm{~Hz}, 2 \mathrm{H}) ;{ }^{13} \mathrm{C}$ NMR $(125 \mathrm{MHz}$, $\left.\mathrm{CDCl}_{3}\right) \delta: 33.76,55.29,64.43,69.37,70.36,70.74,72.38$, 82.42, 89.36, 113.72, 129.38, 133.67, 157.88. HRMS (ESI) calcd for $\mathrm{C}_{20} \mathrm{H}_{18} \mathrm{FeO} 330.0702$, found 330.0712 .

3.2 .5 化合物 $\mathbf{5}$ 的合成方法

氮气保护下，向 $10 \mathrm{~mL}$ Schlenk 反应瓶中，依次加入 $0.5 \mathrm{mmol}$ 化合物 $\mathbf{4}, 1.25 \mathrm{mmol}$ 多聚甲醛, $1.0 \mathrm{mmol}$ 二异 丙胺, $0.4 \mathrm{mmol} \mathrm{CuBr}$, 最后加入无水甲苯 $3 \mathrm{~mL}$, 加毕, 将混合物加热至回流, TLC 监测反应. 反应完全后, 减
压蒸馏蒸去溶剂, 柱层析 $[V($ 石油醚 $): V($ 乙酸乙酯 $)=$ $20:$ 1]纯化得到目标产物 $\mathbf{5 a} \sim \mathbf{5 f}$.

1 '-苯甲基二茂铁联烯 $(\mathbf{5 a})$ ：黄色油状液体，产率 65\%. ${ }^{1} \mathrm{H}$ NMR (500 MHz, $\mathrm{CDCl}_{3}$ ) $\delta: 3.64(\mathrm{~s}, 2 \mathrm{H}), 4.03$ (s, $3 \mathrm{H}), 4.06(\mathrm{~s}, 1 \mathrm{H}), 4.12(\mathrm{~s}, 2 \mathrm{H}), 4.18(\mathrm{~s}, 2 \mathrm{H}), 4.85$ (d, $J=$ $6.5 \mathrm{~Hz}, 1 \mathrm{H}), 5.18(\mathrm{t}, J=6.5 \mathrm{~Hz}, 1 \mathrm{H}), 7.13 \sim 7.15(\mathrm{~m}, 3 \mathrm{H})$, $7.22 \sim 7.25(\mathrm{~m}, 2 \mathrm{H}) ;{ }^{13} \mathrm{C}$ NMR $\left(125 \mathrm{MHz}, \mathrm{CDCl}_{3}\right) \delta$ : $35.61,67.81,68.83,69.12,69.99,77.65,80.41,88.40$, $89.99,126.01,128.37,128.54,141.80,208.94$. HRMS (ESI) calcd for $\mathrm{C}_{20} \mathrm{H}_{18} \mathrm{Fe} 314.0753$, found 314.0753.

1'-(2-氯苯甲基)二茂铁联烯 $(\mathbf{5 b})$ : 黄色油状液体, 产 率 55\%. ${ }^{1} \mathrm{H}$ NMR (500 MHz, $\left.\mathrm{CDCl}_{3}\right) \delta: 3.75(\mathrm{~s}, 2 \mathrm{H}), 4.09$ (s, 1H), $4.13(\mathrm{~s}, 4 \mathrm{H}), 4.19$ (s, 2H), $4.25(\mathrm{~s}, 2 \mathrm{H}), 4.90(\mathrm{~d}$, $J=6.5 \mathrm{~Hz}, 2 \mathrm{H}), 5.84(\mathrm{t}, J=6.5 \mathrm{~Hz}, 1 \mathrm{H}), 7.07 \sim 7.13(\mathrm{~m}$, $3 \mathrm{H}), 7.31(\mathrm{~d}, J=7.0 \mathrm{~Hz}, 1 \mathrm{H}) ;{ }^{13} \mathrm{C} \mathrm{NMR}\left(125 \mathrm{MHz}, \mathrm{CDCl}_{3}\right)$ $\delta: 32.88,67.90,68.93,69.23,70.16,77.61,80.82,86.86$, $89.74,126.72,127.33,129.21,130.10,133.52,139.62$, 208.97. HRMS (ESI) calcd for $\mathrm{C}_{20} \mathrm{H}_{17} \mathrm{ClFe}$ 348.0363, found 348.0359 .

1 '-(3-氯苯甲基)二茂铁联烯 $(\mathbf{5 c})$ : 黄色油状液体, 产 率 50\%. ${ }^{1} \mathrm{H}$ NMR $\left(500 \mathrm{MHz}, \mathrm{CDCl}_{3}\right) \delta: 3.61(\mathrm{~s}, 2 \mathrm{H}), 4.03$ (s, 1H), 4.06 4.08 (m, 3H), $4.14(\mathrm{~s}, 2 \mathrm{H}), 4.20(\mathrm{~s}, 2 \mathrm{H})$, $4.87(\mathrm{~d}, J=6.5 \mathrm{~Hz}, 2 \mathrm{H}), 5.82(\mathrm{t}, J=6.5 \mathrm{~Hz}, 1 \mathrm{H}), 7.02(\mathrm{~d}$, $J=6.5 \mathrm{~Hz}, 1 \mathrm{H}), 7.13 \sim 7.17(\mathrm{~m}, 3 \mathrm{H}) ;{ }^{13} \mathrm{C}$ NMR $(125 \mathrm{MHz}$, $\left.\mathrm{CDCl}_{3}\right) \delta: 35.26,67.78,68.95,69.10,69.92,77.67,80.48$, $87.44,89.82,126.15,126.55,128.48,129.56,134.06$, 143.81, 208.88. HRMS (ESI) calcd for $\mathrm{C}_{20} \mathrm{H}_{17} \mathrm{ClFe}$ 348.0363 , found 348.0365 .

1'-(4-氯苯甲基)二茂铁联烯 (5d): 黄色油状液体, 产 率 58\%. ${ }^{1} \mathrm{H}$ NMR $\left(500 \mathrm{MHz}, \mathrm{CDCl}_{3}\right) \delta: 3.60(\mathrm{~s}, 2 \mathrm{H}), 4.00$ (s, 1H), 4.06 (d, $J=7.5 \mathrm{~Hz}, 3 \mathrm{H}), 4.12$ (s, 2H), 4.19 (s, 2H), $4.86(\mathrm{~d}, J=6.5 \mathrm{~Hz}, 2 \mathrm{H}), 5.81(\mathrm{t}, J=6.5 \mathrm{~Hz}, 1 \mathrm{H}), 7.07$ (t, $J=6.5 \mathrm{~Hz}, 2 \mathrm{H}), 7.20(\mathrm{dd}, J=8.0,5.5 \mathrm{~Hz}, 2 \mathrm{H}) ;{ }^{13} \mathrm{C} \mathrm{NMR}$ $\left(125 \mathrm{MHz}, \mathrm{CDCl}_{3}\right) \delta: 34.91,67.77,68.91,69.08,69.83$, $77.62,80.46,87.80,89.85,128.40,129.70,131.70,140.21$, 208.91. HRMS (ESI) calcd for $\mathrm{C}_{20} \mathrm{H}_{17} \mathrm{ClFe}$ 348.0363, found 348.0370 .

1'-(2-溴苯甲基)二茂铁联烯 (5e)：黄色油状液体，产 率 58\%. ${ }^{1} \mathrm{H}$ NMR $\left(500 \mathrm{MHz}, \mathrm{CDCl}_{3}\right) \delta: 3.72(\mathrm{~s}, 2 \mathrm{H}), 4.16$ (s, 1H), 4.19 (s, 3H), 4.27 (s, 2H), $4.33(\mathrm{~s}, 2 \mathrm{H}), 4.96(\mathrm{~d}$, $J=6.5 \mathrm{~Hz}, 2 \mathrm{H}), 5.82(\mathrm{t}, J=6.5 \mathrm{~Hz}, 1 \mathrm{H}), 7.00 \sim 7.10(\mathrm{~m}$, $2 \mathrm{H}), 7.16(\mathrm{t}, J=6.5 \mathrm{~Hz}, 1 \mathrm{H}), 7.51(\mathrm{~d}, J=7.5 \mathrm{~Hz}, 1 \mathrm{H}) ;{ }^{13} \mathrm{C}$ NMR $\left(125 \mathrm{MHz}, \mathrm{CDCl}_{3}\right) \delta: 35.56,68.16,69.19,69.57$, $70.72,77.58,81.39,87.29,89.72,124.17,127.35,127.57$, 130.14, 132.48, 141.28, 208.71. HRMS (ESI) calcd for 
$\mathrm{C}_{20} \mathrm{H}_{17} \mathrm{BrFe} 391.9859$, found 391.9856 .

1'-(4-甲氧苯甲基)二茂铁联烯 $(\mathbf{5 f})$ : 黄色油状液体, 产率 30\%. ${ }^{1} \mathrm{H}$ NMR (500 MHz, $\left.\mathrm{CDCl}_{3}\right) \delta: 3.59(\mathrm{~s}, 2 \mathrm{H})$, 3.75 (s, 3H), 4.02 (s, 1H), $4.04(\mathrm{~s}, 1 \mathrm{H}), 4.07$ (s, 2H), 4.13 (s, 2H), 4.19 (s, 2H), $4.86(\mathrm{~d}, J=6.5 \mathrm{~Hz}, 2 \mathrm{H}), 5.83(\mathrm{t}, J=$ $6.5 \mathrm{~Hz}, 1 \mathrm{H}), 6.78 \sim 6.81(\mathrm{~m}, 2 \mathrm{H}), 7.08(\mathrm{dd}, J=8.5,5.0 \mathrm{~Hz}$, $2 \mathrm{H}) ;{ }^{13} \mathrm{C} \mathrm{NMR}\left(125 \mathrm{MHz}, \mathrm{CDCl}_{3}\right) \delta: 34.62,55.29,67.70$, 68.71, 69.02, 69.88, 77.53, 80.32, 88.89, 89.91, 113.70, 129.27, 133.96, 157.85, 208.87. HRMS (ESI) calcd for $\mathrm{C}_{21} \mathrm{H}_{20} \mathrm{FeO} 344.0858$, found 344.0864.

辅助材料(Supporting Information) 化合物 $\mathbf{5 a} \sim \mathbf{5 f}$ 的 ${ }^{1} \mathrm{H}$ NMR 和 ${ }^{13} \mathrm{C}$ NMR 谱图. 这些材料可以免费从本刊网 站(http://sioc-journal.cn/)上下载.

\section{References}

[1] Kealy, T. J.; Pauson, P. L. Nature 1951, 168, 1039.

[2] Štěpnička, P. Ferrocenes: Ligands, Materials and Biomolecules, Wiley-VCH, Chichester, U. K., 2008.

[3] Togni, A.; Hayashi, T. Ferrocenes, VCH, New York, 1995

[4] Hildebrandt, A.; Lang, H. Organometallics 2013, 32, 5640.

[5] Fabre, B. Acc. Chem. Res. 2010, 43, 1509.

[6] Musgrave, R. A.; Russell, A. D.; Manners, I. Organometallics 2013, 32, 5654

[7] Dai, L.; Tu, T.; You, S.; Deng, W.; Hou, X. Acc. Chem. Res. 2003, 36,659 .

[8] Atkinson, R. C. J.; Gibson, V. C.; Long, N. J. Chem. Soc. Rev. 2004, 33, 313 .

[9] Schaarschmidt, D.; Lang, H. Organometallics 2013, 32, 5668.

[10] Moriuchi, T.; Hirao, T. Acc. Chem. Res. 2010, 43, 1040.
[11] Braga, S. S.; Silva, A. M. S. Organometallics 2013, 32, 5626.

[12] Hillard, E.; Vessières, A.; Jaouen, G. Top. Organomet. Chem. 2010, 32,81 .

[13] Ornelas, C. New J. Chem. 2011, 35, 1973.

[14] Brasholz, M.; Reissig, H.-U.; Zimmer, R. Acc. Chem. Res. 2009 , 42,45 .

[15] Ma, S. Acc. Chem. Res. 2009, 42, 1679.

[16] Krause, N.; Winter, C. Chem. Rev. 2011, 111, 1994.

[17] Aubert, C.; Fensterbank, L.; Garcia, P.; Malacria, M.; Simonneau, A. Chem. Rev. 2011, 111, 1954

[18] Yu, S.; Ma, S. Angew. Chem., Int. Ed. 2012, 51, 3074

[19] Deng, Y.; Gu, Z.; Ma, S. Chin. J. Org. Chem. 2006, 26, 1468 (in Chinese). (邓友前, 顾振华, 麻生明, 有机化学, 2006, 26, 1468.)

[20] Brandsma, L. Synthesis of Acetylenes, Allenes and Cumulenes, Elsevier, Oxford, 2004.

[21] Krause, N.; Hoffmann-Roder, A. Tetrahedron 2004, 60, 11671.

[22] Brummond, K. M.; Deforrest, J. E. Synthesis 2007, 795.

[23] Ogasawara, M. Tetrahedron: Asymmetry 2009, 20, 259.

[24] Yu, S.; Ma, S. Chem. Commun. 2011, 47, 5384.

[25] Rona, P.; Crabbé, P. J. Am. Chem. Soc. 1969, 91, 3289.

[26] Kuang, J.; Ma, S. J. Org. Chem. 2009, 74, 1763.

[27] Chen, B.; Wang, N.; Fan, W.; Ma, S. Org. Biomol. Chem. 2012, 10, 8465.

[28] Kuang, J.; Ma, S. J. Am. Chem. Soc. 2010, 132, 1786.

[29] Ye, J.; Li, S.; Chen, B.; Fan, W.; Kuang, J.; Liu, J.; Liu, Y.; Miao, B.; Wan, B.; Wang, Y.; Xie, X.; Yu, Q.; Yuan, W.; Ma, S. Org. Lett. 2012, 14, 1346

[30] Kuang, J.; Luo, H.; Ma, S. Adv. Synth. Catal. 2012, 354, 933.

[31] Tang, X.; Zhu, C.; Cao, T.; Kuang, J.; Lin, W.; Ni, S.; Zhang, J.; Ma, S. Nat. Commun. 2013, 4, 2450.

[32] Chen, S.; Shi, J.; Wang, B.; Zhao, H.; Li, B. Appl. Organomet. Chem. 2012, 26, 284

[33] Chen, S.; Wang, B.; Yan, Q.; Shi, J.; Zhao, H.; Li, B. RSC Adv 2013, 3, 1758 . 